\title{
Existence and Well-Posedness for Symmetric Vector Quasi-Equilibrium Problems
}

\author{
Kaihong Wang, Wenyan Zhang, and Min Fang \\ School of Economic Mathematics, Southwestern University of Finance and Economics, Chengdu 610074, China \\ Correspondence should be addressed to Kaihong Wang; wangkaihong@swufe.edu.cn
}

Received 2 February 2014; Accepted 3 June 2014; Published 1 July 2014

Academic Editor: Qamrul Hasan Ansari

Copyright (c) 2014 Kaihong Wang et al. This is an open access article distributed under the Creative Commons Attribution License, which permits unrestricted use, distribution, and reproduction in any medium, provided the original work is properly cited.

\begin{abstract}
An existence result for the solution set of symmetric vector quasi-equilibrium problems that allows for discontinuities is obtained. Moreover, sufficient conditions for the generalized Levitin-Polyak well-posedness of symmetric vector quasi-equilibrium problems are established.
\end{abstract}

\section{Introduction}

Well-posedness of optimization problems was first studied by Tykhonov [1] in 1966. Since then, the notion of wellposedness has been extended to different kinds of optimization problems (see [2-5]). In the book edited by Lucchetti and Revalski [6], Loridan gave a survey on some theoretical results of well-posedness, approximate solutions, and variational principles in vector optimization. Well-posedness for constrained optimization problems was first studied by Levitin and Polyak [7]. The study of Levitin-Polyak wellposedness for convex scalar optimization problems with functional constraints comes from [8]. Recently, this research was extended to general constrained vector optimization problems [9], generalized variational inequality problems with functional constraints [10], and vector equilibrium problems with functional constraints [11].

In 2003, Fu [12] introduced the symmetric vector quasiequilibrium problem (for short (SVQEP)) and studied existence conditions of (SVQEP). (SVQEP) is a generalization of the equilibrium problem, proposed by Blum and Oettli [13], and a unified model of several problems, for example, vector optimization problems, problems of vector Nash equilibria, vector variational inequalities, and vector complementarity problems. Farajzadeh [14] considered existence theorem of the solution of symmetric vector quasi-equilibrium problems in the Hausdorff topological vector space and answered the open question raised by $\mathrm{Fu}$ [12]. In [15], Li et al. obtained existence results for two classes of generalized vector quasiequilibrium problems. Zhang [16] introduced generalized Levitin-Polyak well-posedness for (SVQEP) and obtained sufficient conditions for the generalized Levitin-Polyak wellposedness of (SVQEP).

In this paper, we will introduce existence and wellposed theorem of (SVQEP) for discontinuous vector-valued mapping, which extend the corresponding result in [12] in metric space. Then, by using the conditions of the existence theorem of the solutions to (SVQEP) in [14], we obtain sufficient conditions for the generalized Levitin-Polyak wellposedness of (SVQEP), which improve the result of $[16$, Theorem 4.1].

The paper is organized as follows. In Section 2, we present some preliminary concepts. In Section 3, we prove the existence theorem of (SVQEP) that allows for discontinuities. In Section 4, we obtain generalized Levitin-Polyak well-posed results for (SVQEP).

\section{Preliminaries}

Let $X$ and $Y$ be real locally convex Hausdorff spaces, and let $C$ and $D$ be nonempty subsets of $X$ and $Y$, respectively. Let $Z$ be a real Hausdorff topological vector space and $P \subset Z$ a closed convex and pointed cone with $k_{0} \in$ int $P$.

Assume that $B \subset Z$ is a nonempty subset. A point $b \in B$ is called a minimal point of $B$, if $B \cap(b-P)=\{b\}$. A point $b \in B$ 
is called a weak minimal point of $B$ if $B \cap(b-\operatorname{int} P)=\emptyset$. A point $b \in B$ is called an $\varepsilon$-weak minimal point of $B$ if $B \cap(b-$ $\varepsilon k_{0}-$ int $\left.P\right)=\emptyset$. By $\min B, \min _{w} B$, and $\varepsilon-\min _{w} B$ we denote the sets of all minimal points, weak minimal points, and $\varepsilon$ weak minimal points of $B$, respectively. Obviously, $\min B \subseteq$ $\min _{w} B \subseteq \varepsilon-\min _{w} B$.

Let $E$ be a nonempty subset of $X$ and let $h: E \rightarrow Z$ be a vector-valued mapping. Consider the following vectorvalued optimization problem:

$$
(E, h): \text { minimize } h(x), \quad x \in E .
$$

A point $y \in E$ is called a (weak) minimizer of $(E, h)$ if $h(y)$ is a minimal point (weak minimal point) of the set $h(E)$; namely, for every $x \in E$,

$$
\begin{gathered}
h(x)-h(y) \notin-P \backslash\{0\} \\
(h(x)-h(y) \notin-\operatorname{int} P) .
\end{gathered}
$$

The set of all (weak) minimizers of $(E, h)$ is denoted by $\operatorname{argmin}(E, h)(\operatorname{argwmin}(E, h))$. Obviously, $\operatorname{argmin}(E, h) \subseteq$ $\operatorname{argwmin}(E, h)$.

Let $S: C \times D \rightarrow 2^{C}$ and $T: C \times D \rightarrow 2^{D}$ be two set-valued mappings and let $f, g: C \times D \rightarrow$ $Z$ be two vector-valued mappings. Fu [12] defined a class of symmetric vector quasi-equilibrium problems (for short (SVQEP)), which consist in finding $(\bar{x}, \bar{y}) \in C \times D$ such that $\bar{x} \in S(\bar{x}, \bar{y}), \bar{y} \in T(\bar{x}, \bar{y})$, and

$$
\begin{aligned}
& f(x, \bar{y})-f(\bar{x}, \bar{y}) \notin-\operatorname{int} P, \quad \forall x \in S(\bar{x}, \bar{y}), \\
& g(\bar{x}, y)-g(\bar{x}, \bar{y}) \notin-\operatorname{int} P, \quad \forall y \in T(\bar{x}, \bar{y}) .
\end{aligned}
$$

We call $(\bar{x}, \bar{y})$ a solution of (SVQEP) and denote by $X^{0} \times Y^{0}$ the solution set of (SVQEP). The equilibrium problem contains optimization problem as special case (see $[17,18]$ ). The problem is a generalization of quasi-optimization problem proposed by Crespi and Tan [19].

Definition 1. Let $(Z, P)$ be an ordered topological vector space, let $C$ be a nonempty convex subset of a vector space $X$, and let $h: C \rightarrow Z$ be a vector mapping.

(i) $h$ is called $P$-convex if, for every $x_{1}, x_{2} \in E$ and for every $\lambda \in[0,1]$, one has

$h\left(\lambda x_{1}+(1-\lambda) x_{2}\right) \in \lambda h\left(x_{1}\right)+(1-\lambda) h\left(x_{2}\right)-P$.

(ii) $h$ is called proper $P$-quasiconvex if, for every $x_{1}, x_{2} \in$ $X$ and $\lambda \in[0,1]$, one has either $h\left(\lambda x_{1}+(1-\lambda) x_{2}\right) \epsilon$ $h\left(x_{1}\right)-P$ or $h\left(\lambda x_{1}+(1-\lambda) x_{2}\right) \in h\left(x_{2}\right)-P$.

(iii) $h$ is said to be natural $P$-quasiconvex on $E$ if, for every $x_{1}, x_{2} \in E, \lambda \in[0,1]$, there exists $\mu \in[0,1]$ such that

$$
h\left(\lambda x_{1}+(1-\lambda) x_{2}\right) \in \mu h\left(x_{1}\right)+(1-\mu) h\left(x_{2}\right)-P .
$$

(iv) $h$ is called strict $P$-convex if, for every $x_{1}, x_{2} \in E$ with $x_{1} \neq x_{2}$ and for every $\lambda \in(0,1)$, one has

$$
h\left(\lambda x_{1}+(1-\lambda) x_{2}\right) \in \lambda h\left(x_{1}\right)+(1-\lambda) h\left(x_{2}\right)-\operatorname{int} P .
$$

Remark 2. It is clear that every strict $P$-convex mapping is convex, and every convex or proper $P$-quasiconvex mapping is natural $P$-quasiconvex. A vector mapping may be $P$-convex and not proper $P$-quasiconvex and conversely (see [20]). A vector mapping may be natural $P$-quasiconvex but neither $P$ convex nor proper $P$-quasiconvex.

Let $F: X \rightarrow 2^{Y}$ be a set-valued map. $F$ is said to be upper semicontinuous (u.s.c. for short) at $x \in X$ if, for any open set $U \supset F(x)$, there exists a neighborhood $V$ of $x$ such that

$$
\bigcup_{x \in V} F(x):=F(V) \subset U
$$

If $F$ is u.s.c. at each point of $X$, then $F$ is said to be u.s.c. $F$ is said to be lower semicontinuous (l.s.c. for short) at $x \in X$ if, for any $y \in F(x)$ and any neighborhood $U$ of $y$, there exists a neighborhood $V$ of $x$ such that $\forall x^{\prime} \in V$; we have

$$
F\left(x^{\prime}\right) \cap U \neq \emptyset
$$

$F$ is said to be l.s.c. if $F$ is l.s.c. at every point of $X$. Moreover, $F$ is said to be continuous if $F$ is both l.s.c. and u.s.c. From [21], we can see that $F$ is lower semicontinuous at $x_{0} \in X$ if and only if, for any $y_{0} \in F\left(x_{0}\right)$ and any net $\left\{x_{n}\right\}$ with $x_{n} \rightarrow x_{0}$, there is a net $\left\{y_{n}\right\}$ such that $y_{n} \in F\left(x_{n}\right)$ and $y_{n} \rightarrow y_{0} . F$ is said to be closed if the graph of $F$, that is, $\operatorname{Graph}(F)=\{(x, y): x \in$ $X, y \in F(x)\}$, is a closed set in $X \times Y$.

Let $F: X \rightarrow 2^{X}$ be a set-valued map. A point $x \in X$ is called a fixed point of the set-valued map $F$ if $x \in F(x)$.

Lemma 3. Let $Z$ be a metric space, let $x \in X$, and let $\left\{x_{n}\right\}$ be a sequence converging to $x$ in $X$. Let $a$ and $z$ be two elements in $Z$ such that $z-a \in$ int $P$ and assume that there exist two sequences $\left\{a_{n}\right\}$ and $\left\{z_{n}\right\}$ valued in $Z$ such that

$$
\begin{aligned}
& \lim _{n \rightarrow \infty} d\left(z, z_{n}-P\right)=0, \\
& \lim _{n \rightarrow \infty} d\left(a, a_{n}+P\right)=0,
\end{aligned}
$$

where $d\left(z, z_{n}-P\right)=\min \left\{\left\|z-q_{n}\right\|: q_{n} \in z_{n}-P\right\}$ and $d\left(a, a_{n}+\right.$ $P)=\min \left\{\left\|a-l_{n}\right\|: l_{n} \in a_{n}+P\right\}$. Then for all $n \in N$ sufficiently large one has $z_{n}-a_{n} \in$ int $P$.

The proof of Lemma 3 is similar to that of Lemma 1 in [22]; for details, see [22].

\section{Existence Results for (SVQEP)}

Throughout this section, let $X, Y$, and $Z$ be metric spaces, let the sets $C \subset X$ and $D \subset Y$ be nonempty, convex, and compact subsets, and let $P \subset Z$ be a closed, convex pointed cone with int $P \neq \emptyset$.

Theorem 4. Assume that

(1) $S: C \times D \rightarrow 2^{C}$ and $T: C \times D \rightarrow 2^{D}$ are continuous, and for each $(x, y) \in C \times D, S(x, y), T(x, y)$ are nonempty, closed convex subsets; 
(2) for any $(x, y) \in S(x, y) \times T(x, y),\left(x_{n}, y_{n}\right) \in S\left(x_{n}, y_{n}\right) \times$ $T\left(x_{n}, y_{n}\right)$ for all $n \in N$, and $\left(x_{n}, y_{n}\right)$ converging to $(x, y)$, the following conditions hold:

$$
\begin{aligned}
& d\left(f(x, y), f\left(x_{n}, y_{n}\right)-P\right) \longrightarrow 0 \\
& d\left(g(x, y), g\left(x_{n}, y_{n}\right)-P\right) \longrightarrow 0
\end{aligned}
$$

(3) for any $(x, y) \in S(x, y) \times T(x, y)$ and $\left\{y_{n}\right\}$ converging to $y$ such that $y_{n} \in T\left(x_{n}, y_{n}\right)$, there exists $\bar{x}_{n} \in S\left(x_{n}, y_{n}\right)$ such that $d\left(f(x, y), f\left(\bar{x}_{n}, y_{n}\right)+P\right) \rightarrow 0$; for any $(x, y) \in S(x, y) \times T(x, y)$ and $\left\{x_{n}\right\}$ converging to $x$ such that $x_{n} \in S\left(x_{n}, y_{n}\right)$, there exists $\bar{y}_{n} \in T\left(x_{n}, y_{n}\right)$ such that $d\left(g(x, y), g\left(x_{n}, \bar{y}_{n}\right)+P\right) \rightarrow 0$;

(4) $f(S(x, y), y)$ and $g(x, T(x, y))$ are compact sets;

(5) for any fixed $y \in D, f(x, y)$ is proper $P$-quasiconvex in $x$; for any fixed $x \in C, g(x, y)$ is proper $P$-quasiconvex in $y$.

Then (SVQEP) has a solution.

Proof. Let us define set-valued maps $A: C \times D \rightarrow 2^{C}$ and $B: C \times D \rightarrow 2^{D}$ by

$$
\begin{array}{r}
A(x, y)=\left\{u \in S(x, y) \mid f(u, y) \in \min _{w} f(S(x, y), y)\right\}, \\
\forall(x, y) \in C \times D, \\
B(x, y)=\left\{v \in T(x, y) \mid g(x, v) \in \min _{w} g(x, T(x, y))\right\}, \\
\forall(x, y) \in C \times D .
\end{array}
$$

Similar to the proof of [12, Section 2 Theorem], the set $A(x, y)$ is nonempty and convex. We only need to show the following.

(I) For all $(x, y) \in C \times D$, the set $A(x, y)$ is closed. In fact, let a sequence $\left\{u_{n}\right\} \subset A(x, y)$ and $u_{n} \rightarrow u$; we need to show that $u \in A(x, y)$. It follows from $u_{n} \in S(x, y)$ and the closedness of $S(x, y)$ that $u \in S(x, y)$. Since

$$
f\left(u_{n}, y\right) \in \min _{w} f(S(x, y), y),
$$

we have

$$
f\left(u_{n}, y\right)-f(z, y) \notin \operatorname{int} P, \quad \forall z \in S(x, y) .
$$

If there exists $z_{0} \in S(x, y)$ such that

$$
f(u, y)-f\left(z_{0}, y\right) \in \operatorname{int} P,
$$

from condition $(2), d\left(f(u, y), f\left(u_{n}, y\right)-P\right) \rightarrow 0$; thus,

$$
d\left(f(u, y)-f\left(z_{0}, y\right), f\left(u_{n}, y\right)-f\left(z_{0}, y\right)-P\right) \longrightarrow 0 .
$$

From Lemma $3, f\left(u_{n}, y\right)-f\left(z_{0}, y\right) \in$ int $P$ for $n$ large enough. It is a contradiction.

(II) $A$ is u.s.c., since $C$ is a compact set and $A(x, y) \subset C$. By $[12$, Lemma 2(ii)], we need only to show that the set-valued map $A$ is closed. Let a sequence $\left\{\left(x_{n}, y_{n}\right)\right\} \subset C \times D,\left(x_{n}, y_{n}\right) \rightarrow$ $(x, y)$ and $u_{n} \in A\left(x_{n}, y_{n}\right)$. Since $u_{n} \in S\left(x_{n}, y_{n}\right)$ and the setvalued map $S$ is continuous, we have $u \in S(x, y)$. For any $z \in$ $S(x, y)$, since $S$ is l.s.c., there is a sequence $\left\{z_{n}\right\}, z_{n} \in S\left(x_{n}, y_{n}\right)$ such that $z_{n} \rightarrow z$. Since $f\left(u_{n}, y_{n}\right) \in \min _{w} f\left(S\left(x_{n}, y_{n}\right), y_{n}\right)$, we get

$$
f\left(u_{n}, y_{n}\right)-f\left(z_{n}, y_{n}\right) \notin \operatorname{int} P, \quad \forall z_{n} \in S\left(x_{n}, y_{n}\right) .
$$

If there exists $z \in A(x, y)$ such that $f(u, y)-f(z, y) \in$ int $P$, from (ii),

$$
d\left(f(u, y), f\left(u_{n}, y_{n}\right)-P\right) \longrightarrow 0 .
$$

From (iii), there exists $\bar{z}_{n} \in S\left(x_{n}, y_{n}\right)$ such that

$$
d\left(f(z, y), f\left(\bar{z}_{n}, y_{n}\right)+P\right) \longrightarrow 0 .
$$

By Lemma 3, $f\left(u_{n}, y_{n}\right)-f\left(\bar{z}_{n}, y_{n}\right) \in$ int $P$, which contradicts (16).

Remark 5. It is clear that if $f$ and $g$ are continuous mappings and condition (1) holds, then conditions (2), (3), and (4) of Theorem 4 hold. The following example shows that Theorem 4 improves [12, Section 2. Theorem].

Example 6. Suppose that $X=Y=\mathbb{R}, C=D=[0,2]$, and $P=\mathbb{R}_{+}^{2}$ and let $S: C \times D \rightarrow 2^{C}$ and $T: C \times D \rightarrow 2^{D}$ be defined as $S(x, y)=C$ and $T(x, y)=D$, respectively. For all $(x, y) \in \mathbb{R}^{2}$, let

$$
\begin{aligned}
& f(x, y)= \begin{cases}(0,1), & \text { if } x \in[0,1), \\
(y, 1), & \text { if } x=[1,2],\end{cases} \\
& g(x, y)= \begin{cases}(1,0), & \text { if } y \in[0,1), \\
(1, x), & \text { if } y \in[1,2] .\end{cases}
\end{aligned}
$$

It is clear that the mappings $f$ and $g$ are not continuous, but all the conditions of Theorem 4 hold.

Moreover, let $x^{*}=(1,1)$; we can get from [23, Lemma 2.2] that the mappings $f$ and $g$ are natural $P$-quasiconvex but not demicontinuous (see [14, Definition 2.4]. Therefore, Theorem 4 is different from [14, Theorem 3.1].

\section{Well-Posedness of (SVQEP)}

In this section, we discuss the notion of generalized LevitinPolyak well-posedness for (SVQEP).

Definition 7. A sequence $\left\{\left(x_{n}, y_{n}\right)\right\} \subset C \times D$ is called a Levitin-Polyak approximating solution sequence (in short LP sequence) for (SVQEP) if there exists $\left\{\varepsilon_{n}\right\} \subset \mathbb{R}_{+}$with $\varepsilon_{n} \rightarrow 0$ such that

$$
\begin{gathered}
d\left(x_{n}, S\left(x_{n}, y_{n}\right)\right) \leq \varepsilon_{n}, \quad d\left(y_{n}, T\left(x_{n}, y_{n}\right)\right) \leq \varepsilon_{n}, \\
f\left(x, y_{n}\right)-f\left(x_{n}, y_{n}\right)+\varepsilon_{n} k_{0} \notin-\operatorname{int} P, \quad \forall x \in S\left(x_{n}, y_{n}\right), \\
g\left(x_{n}, y\right)-g\left(x_{n}, y_{n}\right)+\varepsilon_{n} k_{0} \notin-\operatorname{int} P, \quad \forall y \in T\left(x_{n}, y_{n}\right) .
\end{gathered}
$$

Definition 8. The problem (SVQEP) is said to be generalized Levitin-Polyak well-posed (in short LP well-posed) if 
(i) $X^{0} \times Y^{0} \neq \emptyset$;

(ii) for every LP sequence $\left\{\left(x_{n}, y_{n}\right)\right\}$, there exist a subsequence $\left\{\left(x_{n_{k}}, y_{n_{k}}\right)\right\} \subset\left\{\left(x_{n}, y_{n}\right)\right\}$ and an element $\left(x^{*}, y^{*}\right) \in X^{0} \times Y^{0}$ such that $\left(x_{n_{k}}, y_{n_{k}}\right) \rightarrow\left(x^{*}, y^{*}\right)$.

Let us illustrate the notion of generalized LP wellposedness by some examples.

Example 9. Let $X=Y=Z=\mathbb{R}, C=D=[0,1]$, and $P=\mathbb{R}_{+}$. For all $(x, y) \in C \times D$, let $f(x, y)=x+y$ and $g(x, y)=y-x$. Set-valued mappings $S: C \times D \rightarrow 2^{C}$ and $T: C \times D \rightarrow 2^{D}$ are defined by $S(x, y)=T(x, y)=[0,1]$; (SVQEP) is generalized LP well-posed.

Example 10. Let $X=Y=Z=\mathbb{R}, C=D=[0,4 \pi]$, and $P=\mathbb{R}_{+}$. For all $(x, y) \in C \times D$, let $f(x, y)=\sin x y$ and $g(x, y)=\cos x y$. Set-valued mappings $S: C \times D \rightarrow 2^{C}$ and $T: C \times D \rightarrow 2^{D}$ are defined by $S(x, y)=[2 \pi, 4 \pi]$ and $T(x, y)=[1,2]$; (SVQEP) is not generalized LP well-posed.

Remark 11. (i) Generalized LP well-posedness of (SVQEP) implies that the set $X^{0} \times Y^{0}$ is compact.

(ii) It is easy to see that the notion of well-posedness of (SVQEP) generalizes the notion of generalized LP wellposedness of vector equilibrium problem introduced in [20].

Theorem 12. Let $\left\{\varepsilon_{n}\right\} \subset \mathbb{R}_{+}$with $\varepsilon_{n} \rightarrow 0$. Under the assumptions of Theorem 4, (SVQEP) is generalized LP wellposed.

Proof. Let $\left\{\varepsilon_{n}\right\} \subset \mathbb{R}_{+}$with $\varepsilon_{n} \rightarrow 0$ and

$$
\begin{array}{r}
d\left(x_{n}, S\left(x_{n}, y_{n}\right)\right) \leq \varepsilon_{n}, \\
f\left(x, y_{n}\right)-f\left(x_{n}, y_{n}\right)+\varepsilon_{n} k_{0} \notin-\operatorname{int} P, \\
\forall x \in S\left(x_{n}, y_{n}\right) ; \\
d\left(y_{n}, T\left(x_{n}, y_{n}\right)\right) \leq \varepsilon_{n}, \\
g\left(x_{n}, y\right)-g\left(x_{n}, y_{n}\right)+\varepsilon_{n} k_{0} \notin-\operatorname{int} P, \\
\forall y \in T\left(x_{n}, y_{n}\right) .
\end{array}
$$

Since $S, T$ are continuous and compact-valued, there exist a subsequence $\left\{\left(x_{n_{k}}, y_{n_{k}}\right)\right\} \subset\left\{\left(x_{n}, y_{n}\right)\right\}$ and an element $\left(x_{0}, y_{0}\right) \in S\left(x_{0}, y_{0}\right) \times T\left(x_{0}, y_{0}\right)$ such that $\left\{\left(x_{n_{k}}, y_{n_{k}}\right)\right\} \rightarrow$ $\left(x_{0}, y_{0}\right)$. If there exists $\bar{x} \in S\left(x_{0}, y_{0}\right)$, such that $f(\bar{x}, y) \in$ $f\left(x_{0}, y_{0}\right)-$ int $P$, from Theorem 4 (ii),

$$
d\left(f\left(x_{0}, y_{0}\right), f\left(x_{n_{k}}, y_{n_{k}}\right)-\varepsilon_{n_{k}} k_{0}-P\right) \longrightarrow 0
$$

From Theorem 4(iii), there exists $\bar{x}_{n_{k}} \in S\left(x_{n_{k}}, y_{n_{k}}\right)$ such that

$$
d\left(f(\bar{x}, y), f\left(\bar{x}_{n_{k}}, y_{n_{k}}\right)+P\right) \longrightarrow 0 \text {. }
$$

By Lemma 3,

$$
f\left(\bar{x}_{n_{k}}, y_{n_{k}}\right)-f\left(x_{n_{k}}, y_{n_{k}}\right)+\varepsilon_{n_{k}} k_{0} \in-\operatorname{int} P
$$

when $n_{k}$ is large enough, which is a contradiction. Therefore,

$$
f\left(x, y_{0}\right)-f\left(x_{0}, y_{0}\right) \notin-\operatorname{int} P, \quad \forall x \in S\left(x_{0}, y_{0}\right) \text {. }
$$

Similarly,

$$
g\left(x_{0}, y\right)-g\left(x_{0}, y_{0}\right) \notin-\operatorname{int} P, \quad \forall y \in T\left(x_{0}, y_{0}\right) .
$$

From Definition 7, (SVQEP) is generalized LP well-posed.

Definition 13 (see [23]). Let $X$ be a topological space, and let $Z$ be a topological vector space. A function $f: X \rightarrow Z$ is said to be demicontinuous if

$$
f^{-1}(M)=\{x \in X: f(x) \in M\}
$$

is closed in $X$ for each closed half space $M \subset Z$.

Lemma 14 (see [23]). Let $X$ be a topological space, $Z$ a topological vector space, and $f: X \rightarrow Z$ a demicontinuous function. Then for any $x^{*} \in Z^{*}$, the composite function $x^{*} \circ f$ is continuous, where $Z^{*}$ is the topological dual space of $Z$.

Let $P \subset Z$ be a closed convex and pointed cone with int $P \neq \emptyset$ and

$$
T=\left\{x^{*} \in Z^{*}: x^{*} \text { separates the sets } P \text { and }-\operatorname{int} P\right\} .
$$

By [24, p. 165, Theorem 2], there exists $x^{*} \in Z^{*}$ such that

$$
\begin{gathered}
\forall x \in P, \quad x^{*}(x) \geq 0, \\
\forall x \in-\operatorname{int} P, \quad x^{*}(x)<0 .
\end{gathered}
$$

We get that $T \neq \emptyset$.

Lemma 15. If $\left(x^{*}, x\right)<0$ for all $x^{*} \in T$, then $x \in-\operatorname{int} P$.

Proof. If we assume that there exists $x \notin-$ int $P$ such that $\left(x^{*}, x\right)<0$ for all $x^{*} \in T$, then we have

$$
\text { - int } P \bigcap\{\lambda x+(1-\lambda) p: \lambda \in[0,1], p \in P\}=\emptyset .
$$

If not, there exist $y \in \operatorname{int} P, \lambda \in[0,1]$, and $p \in P$ such that $-y \in \lambda x+(1-\lambda) p$. Thus,

$$
x=-\frac{1}{\lambda}\left(\lambda \cdot \frac{y}{\lambda}+(1-\lambda) p\right) \in-\operatorname{int} P .
$$

It is a contradiction. Thus, (30) holds. By [24, p. 165, Theorem 2], there exists $x^{* \prime} \in Z^{*}$ such that, for all $y \in-\operatorname{int} P$,

$$
x^{* \prime}(y)<0
$$

and for all $y \in\{\lambda x+(1-\lambda) p: \lambda \in[0,1], p \in P\}$,

$$
x^{* \prime}(y) \geq 0 \text {. }
$$

Then, $x^{* \prime}(x) \geq 0$ and $x^{* \prime} \in T$. This, however, contradicts the fact that $\left(x^{*}, x\right)<0$ for all $x^{*} \in T$. 
Theorem 16. Assume that

(1) $S: C \times D \rightarrow 2^{C}$ and $T: C \times D \rightarrow 2^{D}$ are continuous, and for each $(x, y) \in C \times D, S(x, y), T(x, y)$ are nonempty, closed convex subsets;

(2) $f, g: C \times D \rightarrow Z$ are demicontinuous;

(3) for any fixed $y \in D, f(x, y)$ is natural P-quasiconvex in $x$; for any fixed $x \in C, g(x, y)$ is natural $P$ quasiconvex in $y$.

Then (SVQEP) is generalized LP well-posed.

Proof. From [14, Theorem 3.1], $X^{0} \times Y^{0} \neq \emptyset$. Let $\left\{\varepsilon_{n}\right\} \subset \mathbb{R}_{+}$ with $\varepsilon_{n} \rightarrow 0$ and

$$
\begin{array}{r}
d\left(x_{n}, S\left(x_{n}, y_{n}\right)\right) \leq \varepsilon_{n}, \\
f\left(x, y_{n}\right)+\varepsilon_{n} k_{0}-f\left(x_{n}, y_{n}\right) \notin-\operatorname{int} P, \\
\forall x \in S\left(x_{n}, y_{n}\right) ; \\
d\left(y_{n}, T\left(x_{n}, y_{n}\right)\right) \leq \varepsilon_{n}, \\
g\left(x_{n}, y\right)+\varepsilon_{n} k_{0}-g\left(x_{n}, y_{n}\right) \notin-\operatorname{int} P, \\
\forall y \in T\left(x_{n}, y_{n}\right) .
\end{array}
$$

Since $S, T$ are continuous and compact-valued, there exist a subsequence $\left\{\left(x_{n_{k}}, y_{n_{k}}\right)\right\} \subset\left\{\left(x_{n}, y_{n}\right)\right\}$ and an element $\left(x_{0}, y_{0}\right) \in S\left(x_{0}, y_{0}\right) \times T\left(x_{0}, y_{0}\right)$ such that $\left(x_{n_{k}}, y_{n_{k}}\right) \rightarrow$ $\left(x_{0}, y_{0}\right)$. If there exists $\bar{x} \in S\left(x_{0}, y_{0}\right)$, such that

$$
f\left(\bar{x}, y_{0}\right) \in f\left(x_{0}, y_{0}\right)-\operatorname{int} P,
$$

for all $x^{*} \in T$, we have

$$
x^{*}\left(f\left(\bar{x}, y_{0}\right)-f\left(x_{0}, y_{0}\right)\right)<0 .
$$

Then,

$$
x^{*}\left(f\left(\bar{x}, y_{0}\right)\right)<x^{*}\left(f\left(x_{0}, y_{0}\right)\right) .
$$

Since $S$ is l.s.c. at $\bar{x}$, there exists $\bar{x}_{n} \in S\left(x_{n}, y_{n}\right)$ such that $\bar{x}_{n} \rightarrow$ $\bar{x}$. By Lemma 14, $x^{*}\left(f\left(\bar{x}_{n_{k}}, y_{n_{k}}\right)\right)+x^{*}\left(\varepsilon_{n_{k}} k_{0}\right)<x^{*}\left(f\left(x_{n_{k}}, y_{n_{k}}\right)\right)$ when $n_{k}$ is large enough. By Lemma 15 ,

$$
f\left(\bar{x}_{n_{k}}, y_{n_{k}}\right)-f\left(x_{n_{k}}, y_{n_{k}}\right)+\varepsilon_{n_{k}} k_{0} \in-\operatorname{int} P,
$$

which contradicts (34). Therefore, (SVQEP) is generalized LP well-posed.

Let $K \subset \mathbb{R}^{n}$ be a closed set, let $f: K \subseteq \mathbb{R}^{n} \rightarrow \mathbb{R}^{l}$, and consider a cone $C \subseteq \mathbb{R}^{l}$ to be closed, convex, pointed, and with nonempty interior. We consider the following vector optimization problem.

(VP) Find $x_{0} \in K$ such that

$$
f(x)-f\left(x_{0}\right) \notin-\operatorname{int} C, \quad \forall x \in K .
$$

As a consequence of Theorem 16, we have the following well-posed result for the solution of (VP).
Corollary 17. Let $K \subset \mathbb{R}^{n}$ be a compact set, and let $f: \mathbb{R}^{n} \rightarrow$ $\mathbb{R}^{l}$ be demicontinuous and natural $C$-quasiconvex on $K$. Then, $(V P)$ is generalized LP well-posed.

Remark 18. (i) From Remark 2, every strict $P$-convex mapping is natural $P$-quasiconvex. Then Theorem 16 improves [16, Theorem 4.1].

(ii) From [23, Lemma 2.1(iii)], every natural $C$ quasiconvex function is $C$-quasiconvex. In the assumption of $f$ being continuous, Corollary 17 is a special case of $[18$, Theorem 4.2].

\section{Conflict of Interests}

The authors declare that there is no conflict of interests regarding the publication of this paper.

\section{Acknowledgments}

The authors would like to thank the anonymous referee for his/her valuable comments and suggestions, which helped in improving the paper. This work is supported by the Fundamental Research Funds for the Central Universities (JBK130401), by Humanities and Social Science Project of Ministry of Education of China (14XJC), and by Soft Science Research Project of Sichuan Province (2014ZR0027).

\section{References}

[1] A. N. Tykhonov, "On the stability of the functional optimization problem," USSR Computational Mathematics and Mathematical Physics, vol. 6, no. 4, pp. 28-33, 1966.

[2] E. Miglierina, E. Molho, and M. Rocca, "Well-posedness and scalarization in vector optimization," Journal of Optimization Theory and Applications, vol. 126, no. 2, pp. 391-409, 2005.

[3] D. Dentcheva and S. Helbig, "On variational principles, level sets, well-posedness, and $\varepsilon$-solutions in vector optimization," Journal of Optimization Theory and Applications, vol. 89, no. 2, pp. 325-349, 1996.

[4] R. Hu and Y.-P. Fang, "Set-valued increasing-along-rays maps and well-posed set-valued star-shaped optimization," Journal of Mathematical Analysis and Applications, vol. 331, no. 2, pp. 13711383, 2007.

[5] M. B. Lignola, "Well-posedness and L-well-posedness for quasivariational inequalities," Journal of Optimization Theory and Applications, vol. 128, no. 1, pp. 119-138, 2006.

[6] R. Lucchetti and I. Revalski, Eds., Recent Developments in Well-Posed Variational Problems, vol. 331, Kluwer Academic Publishers, Dordrecht, The Netherlands, 1995.

[7] E. S. Levitin and B. T. Polyak, "Convergence of minimizing sequences in conditional extremum problems," Soviet Mathematics Doklady, vol. 7, pp. 764-767, 1966.

[8] A. S. Konsulova and J. P. Revalski, "Constrained convex optimization problems-well-posedness and stability," Numerical Functional Analysis and Optimization, vol. 15, no. 7-8, pp. 889907, 1994.

[9] X. X. Huang and X. Q. Yang, "Levitin-Polyak well-posedness of constrained vector optimization problems," Journal of Global Optimization, vol. 37, no. 2, pp. 287-304, 2007. 
[10] X. X. Huang and X. Q. Yang, "Levitin-Polyak well-posedness in generalized variational inequality problems with functional constraints," Journal of Industrial and Management Optimization, vol. 3, no. 4, pp. 671-684, 2007.

[11] S. J. Li and M. H. Li, "Levitin-Polyak well-posedness of vector equilibrium problems," Mathematical Methods of Operations Research, vol. 69, no. 1, pp. 125-140, 2009.

[12] J.-Y. Fu, "Symmetric vector quasi-equilibrium problems," Journal of Mathematical Analysis and Applications, vol. 285, no. 2, pp. 708-713, 2003.

[13] E. Blum and W. Oettli, "From optimization and variational inequalities to equilibrium problems," The Mathematics Student, vol. 63, no. 1-4, pp. 123-145, 1994.

[14] A. P. Farajzadeh, "On the symmetric vector quasi-equilibrium problems," Journal of Mathematical Analysis and Applications, vol. 322, no. 2, pp. 1099-1110, 2006.

[15] S. J. Li, K. L. Teo, and X. Q. Yang, "Generalized vector quasiequilibrium problems," Mathematical Methods of Operations Research, vol. 61, no. 3, pp. 385-397, 2005.

[16] W. Y. Zhang, "Well-posedness for convex symmetric vector quasi-equilibrium problems," Journal of Mathematical Analysis and Applications, vol. 387, no. 2, pp. 909-915, 2012.

[17] G. P. Crespi, M. Papalia, and M. Rocca, "Extended wellposedness of quasiconvex vector optimization problems," Journal of Optimization Theory and Applications, vol. 141, no. 2, pp. 285-297, 2009.

[18] G. P. Crespi, A. Guerraggio, and M. Rocca, "Well posedness in vector optimization problems and vector variational inequalities," Journal of Optimization Theory and Applications, vol. 132, no. 1, pp. 213-226, 2007.

[19] G. P. Crespi and N. X. Tan, "On vector quasi-optimization problems," Rendiconti del Seminario Matematico di Messina II, vol. 8(23), pp. 283-296, 2001.

[20] F. Ferro, "A minimax theorem for vector-valued functions," Journal of Optimization Theory and Applications, vol. 60, no. 1, pp. 19-31, 1989.

[21] J.-P. Aubin and I. Ekeland, Applied Nonlinear Analysis, John Wiley \& Sons, New York, NY, USA, 1984.

[22] P. Loridan and J. Morgan, "Convergence of approximate solutions and values in parametric vector optimization," in Vector Variational Inequalities and Vector Equilibria, F. Giannessi, Ed., vol. 38, pp. 335-350, Kluwer Academic Publishers, Dordrecht, The Netherlands, 2000.

[23] T. Tanaka, "Generalized quasiconvexities, cone saddle points, and minimax theorem for vector-valued functions," Journal of Optimization Theory and Applications, vol. 81, no. 2, pp. 355-377, 1994.

[24] C. Swartz, An Introduction to Functional Analysis, vol. 157, Marcel Dekker, New York, NY, USA, 1992. 


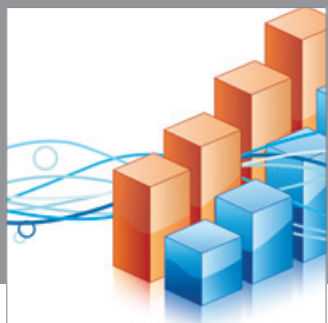

Advances in

Operations Research

mansans

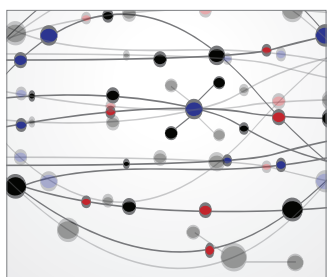

The Scientific World Journal
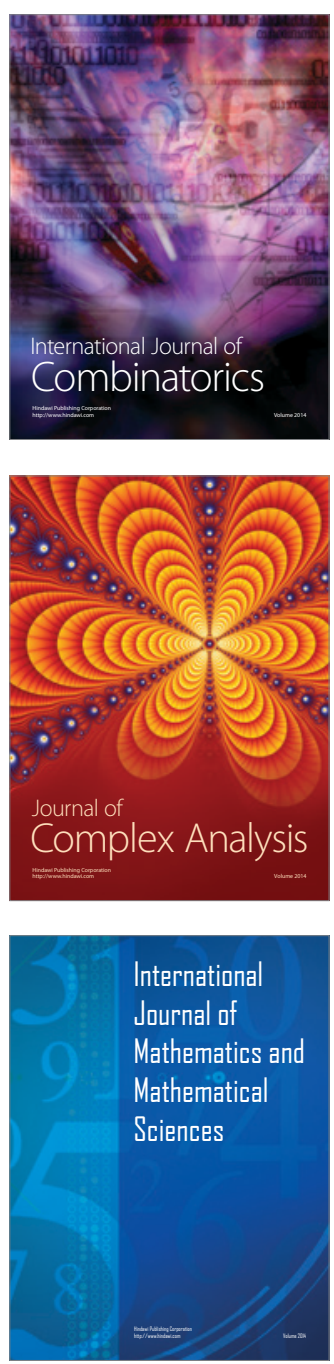
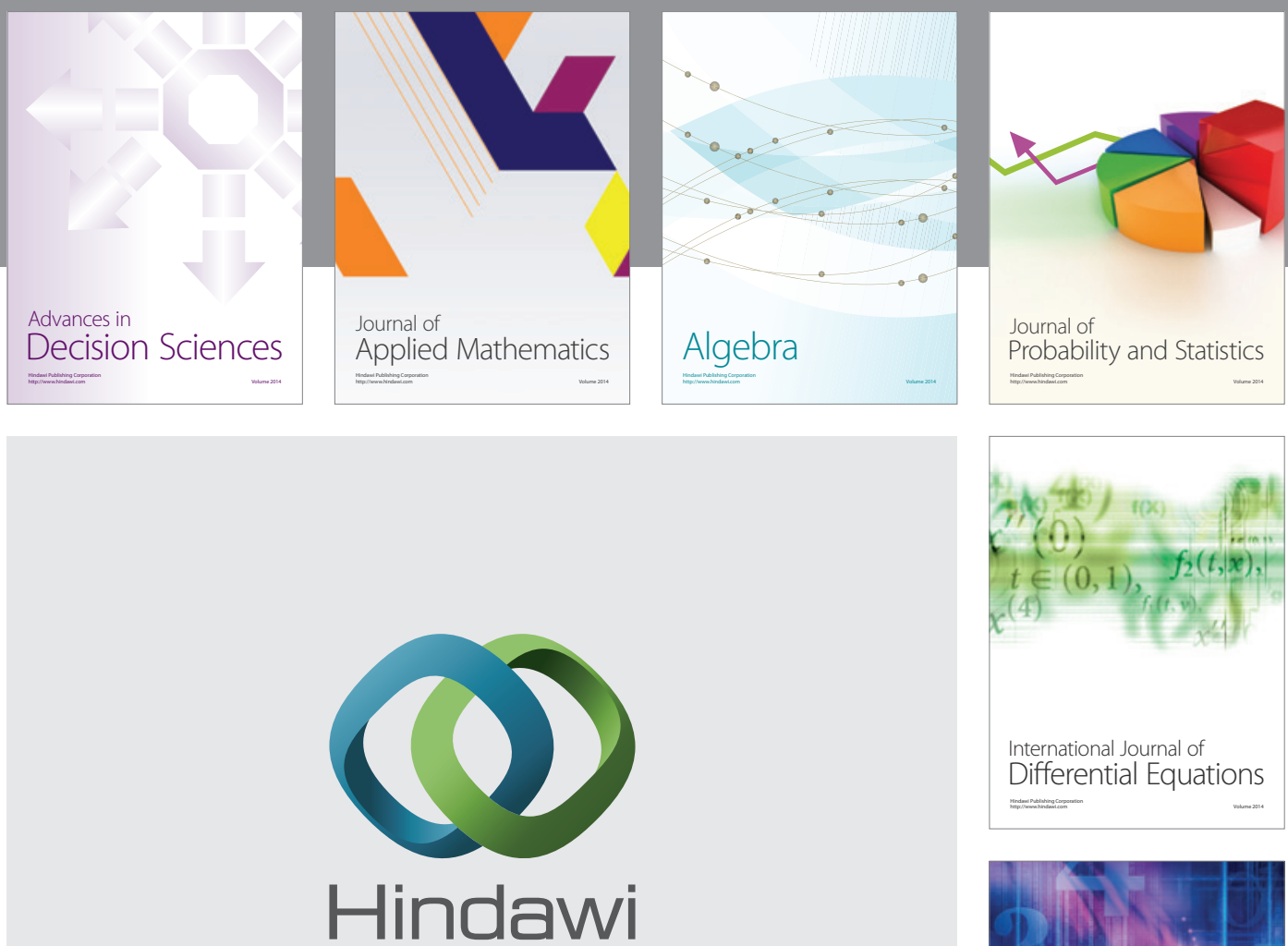

Submit your manuscripts at http://www.hindawi.com
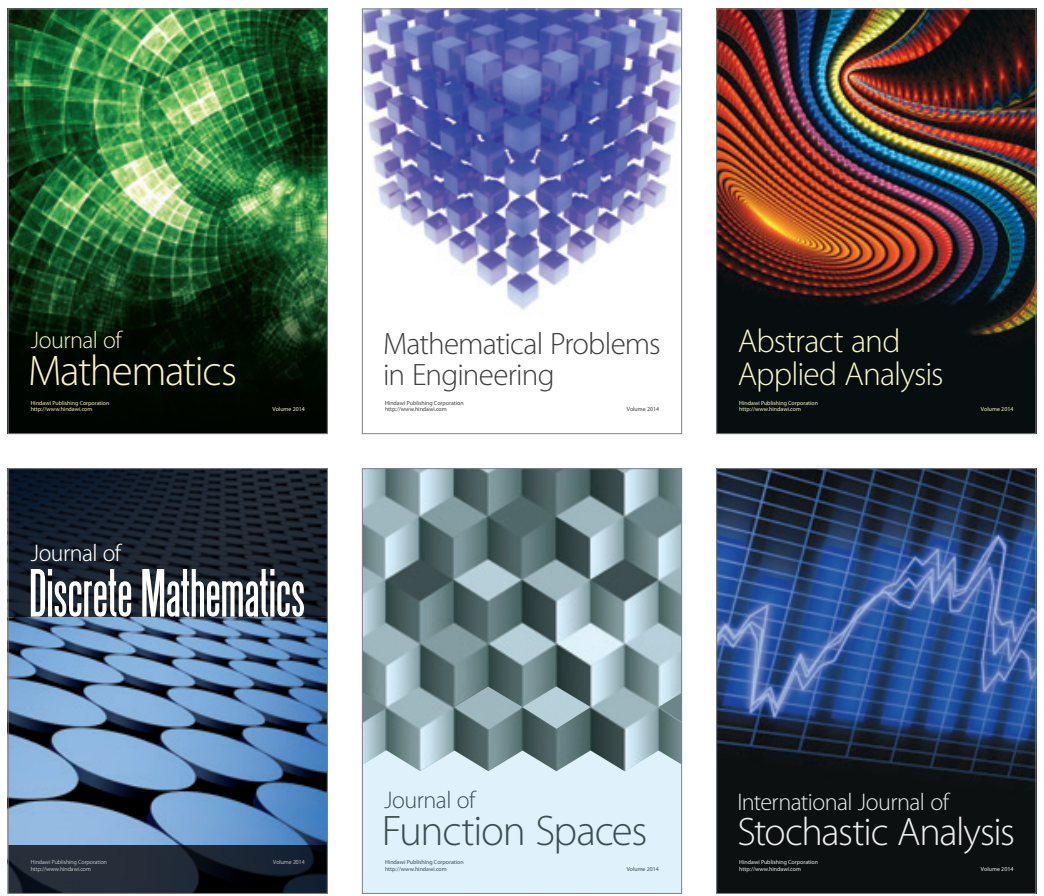

Journal of

Function Spaces

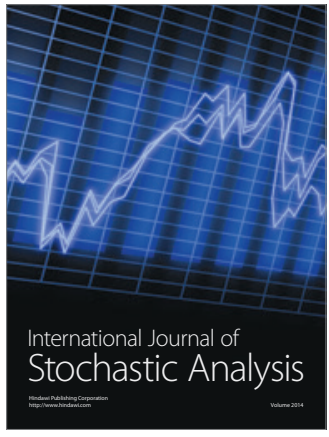

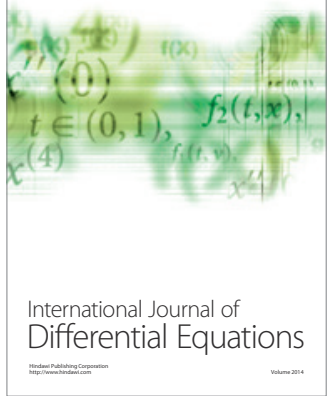
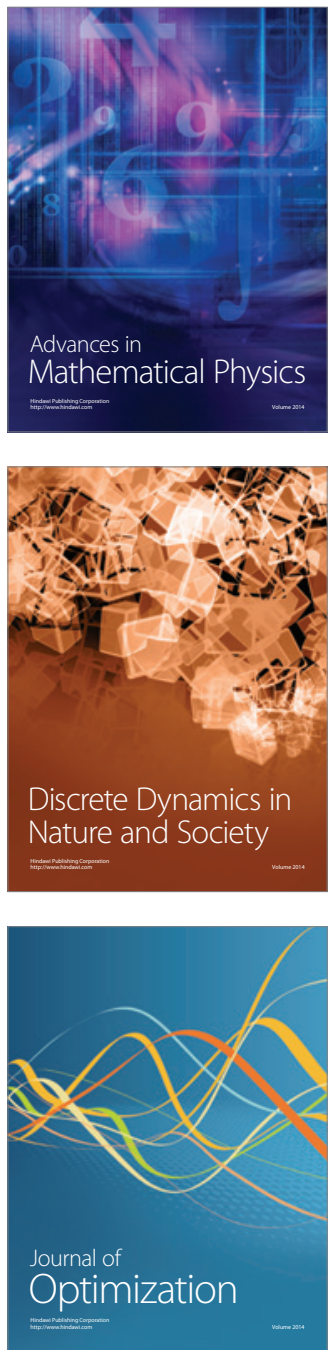